\title{
Berry phase for ferromagnet with fractional spin
}

\author{
A. G. Abanov \\ Department of Physics and Astronomy, Stony Brook University, Stony Brook, NY 11794-3800 \\ Ar. Abanov \\ Los Alamos National Laboratory, Theoretical division, MS262, Los Alamos, NM 87545
}

\begin{abstract}
We study the double exchange model on two lattice sites with one conduction electron in the limit of an infinite Hund's interaction. While this simple problem is exactly solvable, we present an approximate solution which is valid in the limit of large core spins. This solution is obtained by integrating out charge degrees of freedom. The effective action of two core spins obtained in the result of such an integration resembles the action of two fractional spins. We show that the action obtained via naive gradient expansion is inconsistent. However, a "non-perturbative" treatment leads to an extra term in the effective action which fixes this inconsistency. The obtained "Berry phase term" is geometric in nature. It arises from a geometric constraint on a target space imposed by an adiabatic approximation.
\end{abstract}

\section{INTRODUCTION}

A double exchange model was introduced in [1, 2 to describe the motion of conduction electrons in the background of magnetic ions. In its simplest form the Hamiltonian for the model can be written as:

$$
H=-\sum_{\langle i j\rangle}\left(t c_{i}^{\dagger} c_{j}+\text { h.c. }\right)-\sum_{i} J_{H} \vec{s}_{i} \vec{S}_{i},
$$

where $c_{i}$ is the electron annihilation operator on the site $i$ of the lattice, $\vec{s}_{i}=\frac{1}{2} c_{i}^{\dagger} \vec{\sigma} c_{i}$ is a spin $1 / 2$ of an electron [12] at site $i, \vec{S}_{i}$ is a core spin. The first term of (11) describes the hopping of conduction electrons between nearest neighbor sites $i$ and $j$ of the lattice. The hopping amplitude is $t$. The second term is a ferromagnetic Hund's interaction between the core spin and the spin of the conduction electron at the same lattice site. To specify the model completely we have to fix the total number of conduction electrons in the sample. We denote filling factor $x=\sum_{i} c_{i}^{\dagger} c_{i} / N$, where $N$ is a total number of sites of the lattice. In this paper we are interested [3] in the limit of a very strong Hund's interaction $J_{H} / t \rightarrow \infty$ and of very large core spins $S \gg 1$. In (11) we did not include any (bare) core spin interactions, e.g., due to superexchange. The only interaction between core spins in the model (1) occurs through the exchange by conduction electrons.

Because of an infinite Hund's coupling $J_{H}$ an electron spin is always aligned with a core spin on any given lattice site. Therefore, the effective hopping amplitude of an electron between two nearest neighbors is the largest when corresponding core spins are parallel. In this case an electron can hop between lattice sites without changing its spin orientation. One concludes that conduction electrons induce a ferromagnetic interaction between core spins [2, 6].

In the limit of large core spins $S \gg 1$ we consider one can use an adiabatic approximation and think of core spins as of slow variables so that the conduction electrons (fast variables) are always in the ground state in the background of slowly moving core spins. It is tempting to average over the motion of conduction electrons (to integrate conduction electrons out) and obtain an effective spin model describing the dynamics of core spins (and electron spins) induced by charge degrees of freedom 13. In an almost ferromagnetic state the average spin per lattice site is $S+\frac{1}{2} x$ corresponding to the sum of a core spin and an average electron spin. Therefore, heuristically, one arrives to the model of ferromagnetically interacting fractional spins. However, this immediately raises the question of what one means by a fractional spin. Although the average spin per lattice site is fractional, the total spin operator on a given site may take only values $S$ (no electron on the site) or $S+1 / 2$ (there is an electron on the site). Moreover, a fractional value of $2 S$ is incompatible with quantum mechanics of spins (see section [I]). Resolving this apparent controversy is a main goal of this paper.

The paper is organized in the following way. The aim of the section [II is mainly pedagogical. We establish notations and remind the reader how to write the path integral for a single spin. We show how the ambiguity of the Berry phase for a single spin requires $2 S$ be an integer. For completeness we derive the Berry phase for a single spin in Appendix A using a fermionic determinant representation for the action. The reader familiar with the subject can start from the section III where we consider the double exchange model (11) in the limit $J_{H} / t \rightarrow \infty$ and $S \gg 1$. In section IV we formulate a double exchange model on two core spins and obtain our main result - an effective action for core spins induced by a single conduction electron. This action (namely, its kinetic part) answers the question of how to write Berry phase for a system of (two) fractional spins. Appendix B contains more accurate calculation of the effective action which uses the method of gradient expansion. We discuss the topology behind Berry phase for fractional spins in section $\mathrm{V}$ and conclude in section $\mathrm{VI}$. 


\section{PATH INTEGRAL FOR SINGLE SPIN}

In this section we remind the reader how to write down the path integral for a single quantum spin and discuss topological reasons for spin quantization.

Quantum spin $\vec{S}$ is defined as a representation of dimension $2 S+1$ of an $S U(2)$ Lie algebra with commutation relations

$$
\begin{aligned}
{\left[S^{+}, S^{-}\right] } & =2 S^{z}, \\
{\left[S^{z}, S^{ \pm}\right] } & = \pm S^{ \pm},
\end{aligned}
$$

where as usual $S^{ \pm}=S^{x} \pm i S^{y}$. An $S U(2)$ Lie algebra has irreducible finite dimensional representations of dimension $2 S+1$ labelled by spin $S$ taking integer or half-integer values so that $\vec{S}^{2}=S(S+1)$. We would like to construct the classical action for the time dependent unit vector $\vec{n}(t)$ which upon quantization by path integral reproduces (2). One could start directly from (2) and construct the path integral using coherent states corresponding to an $S U(2)$ group (see e.g., Ref.[7]). In Appendix A we give an alternative derivation of this path integral using the fermionic determinant representation.

The result is given by partition function

$$
Z=\int D \vec{n} e^{-W_{E}[\vec{n}]},
$$

where

$$
W_{E}[\vec{n}]=i(2 S) 2 \pi \int_{\mathcal{B}} d^{2} x \frac{1}{8 \pi} \epsilon^{a b c} \epsilon_{\mu \nu} n^{a} \partial_{\mu} n^{b} \partial_{\nu} n^{c} .
$$

Let us explain what (3,4) mean. First of all we used an Euclidean time representation replacing real physical time $t$ by an imaginary time $\tau=i t$. The change to an imaginary time changes quantum amplitude $e^{i W}$ used in path integral into a "Boltzmann" factor $e^{-W_{E}}$. We impose periodic boundary conditions (in imaginary time) on $\vec{n}(\tau)$ so that $\vec{n}(\beta)=\vec{n}(0)$ and $\beta$ is a maximal span of an imaginary time. With these boundary conditions one can think of time as of a one-dimensional circle with identification of point $\tau=\beta$ with $\tau=0$. Physical results will be obtained in the limit $\beta \rightarrow \infty$ after an analytical continuation back to the real time $t=-i \tau$. Imaginary time representation is slightly more convenient for calculations and for a discussion of topological aspects of spin quantization.

Secondly, the two-dimensional integration in (4) is performed over some closed domain $\mathcal{B}$ of two dimensional plane such that the boundary of this domain is identified with an imaginary time. The values of $\vec{n}$ field in the area $\mathcal{B}$ are defined as an extension from the physical trajectory $\vec{n}(\tau)$ so that $\vec{n}(\tau, u)$ is a smooth function of $\tau \in[0, \beta]$ and auxiliary variable $u \in[0,1]$ such that $\vec{n}(\tau, u=0)=(0,0,1)$ and $\vec{n}(\tau, u=1)=\vec{n}(\tau)$. We shall refer to the action (4) as to a Berry phase \& for a spin (see Appendix A). Although the action (4) is given in terms of two-dimensional integral over auxiliary space, the variation of the integrand is a full derivative. Therefore, the variation of Berry phase (4) is given by a one-dimensional integral over the "physical" (imaginary) time as

$$
\delta W_{E}=i S \int_{0}^{\beta} d \tau \epsilon^{a b c} \delta n^{a} n^{b} \partial_{\tau} n^{c}
$$

We see that the variation of (4) does not depend on the choice of an extension of $\vec{n}(\tau)$ in the unphysical domain $\mathcal{B}$. Using (5) one can show that the equation $\delta\left(W_{E}+W_{E}^{h}\right)=$ 0 , where $W_{E}^{h}=\int_{0}^{\beta} d \tau S \vec{h} \vec{n}$ is a coupling to an external magnetic field gives a classical equation of motion $i \partial_{\tau} \vec{n}=$ $[\vec{h} \times \vec{n}]$. The latter is the equation of precession of spin in magnetic field $\vec{h}$.

Partition function (3) with the action (4) solve the problem of path integral representation for the algebra (2). Namely, the commutation relations (2) can be obtained as a result of quantization [7] of "classical action" (4).

\section{A. Berry phase and quantization of spin}

We have already checked that an infinitesimal variation of Berry phase $\delta W_{E}[\vec{n}]$ does not depend on the extension of $\vec{n}(\tau)$ into the auxiliary domain $\mathcal{B}$. How about $W_{E}[\vec{n}]$ itself? One can easily show (see Appendix A) that the action (4) is equal to $i(2 S) \Omega / 2$ where $\Omega$ is a solid angle swept by a unit vector $\vec{n}$ during its motion (see Fig. 11). This angle is not uniquely defined: one can always add or subtract any angle which is a multiple of a full solid angle $4 \pi$.

Therefore, $W_{E}[\vec{n}]$ is not unambiguously defined and is a multi-valued functional of $\vec{n}(\tau)$. Multi-valued functionals of this type were studied in both mathematics and quantum field theory (see e.g., [9]) and are often referred to as WZNW term - Wess-Zumino-NovikovWitten term. Although $W_{E}(\vec{n})$ is a multiple-valued functional, in partition function (3) one needs $e^{-W_{E}[\vec{n}]}$ which is a single-valued functional of $\vec{n}(\tau)$ under the condition that the coefficient $2 S$ is an integer. Then $e^{-\delta W_{E}}=e^{-i(2 S) 2 \pi k}=1$ and the Boltzmann weights $e^{-W_{E}(\vec{n})}$ is defined unambiguously by physical configurations $\vec{n}(\tau)$. One can think of this constraint on the value of $2 S$ as of the reason for spin to be an integer or a half-integer. Having non-integer $2 S$ in (4) amounts to having inconsistent theory.

\section{B. $C P^{1}$ representation of Berry phase and topology}

There is a one more representation of Berry phase which is particularly convenient for the purposes of this paper. It is a so-called $C P^{1}$ representation. We write $\vec{n}=$ $z^{\dagger} \vec{\sigma} z$, where $z=\left(z_{1}, z_{2}\right)^{t}$ is a complex two-component vector satisfying the constraint $z^{\dagger} z=\left|z_{1}\right|^{2}+\left|z_{2}\right|^{2}=1$. The latter reproduces $\vec{n}^{2}=1$. 


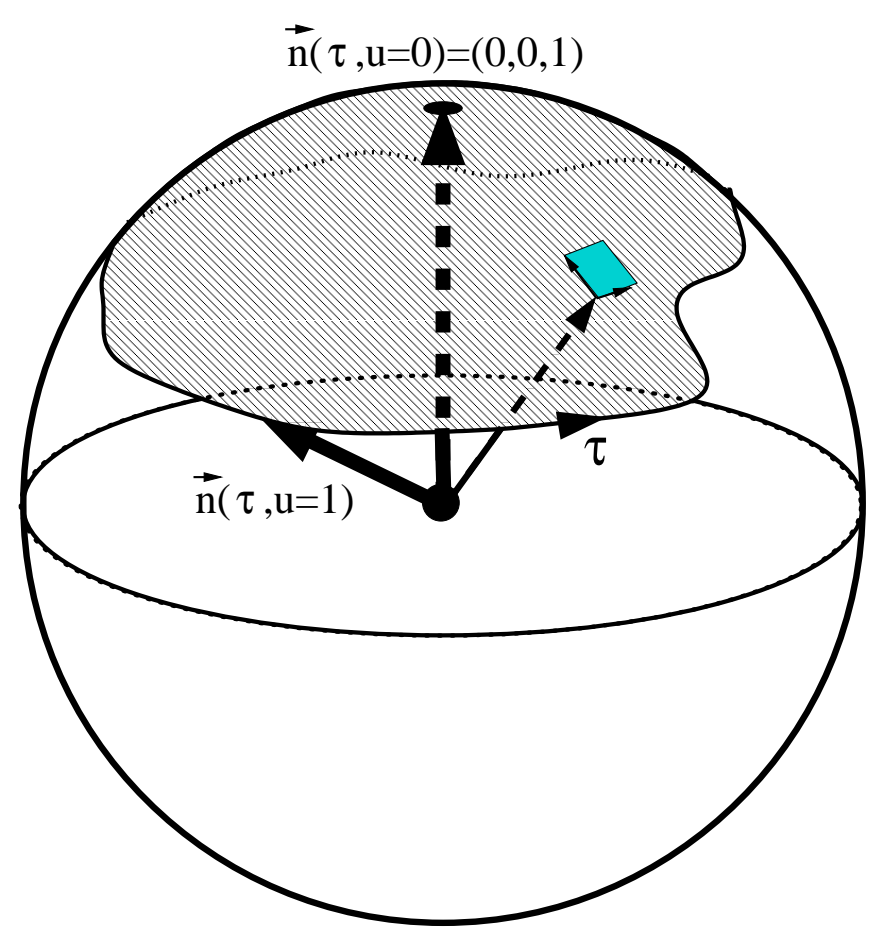

FIG. 1: The unit vector $\vec{n}(\tau)$ draws a closed line on the surface of a sphere with unit radius during its motion in imaginary time. Berry phase is proportional to the solid angle (shaded region) swept by the vector $\vec{n}(\tau)$. One can calculate this solid angle by extending $\vec{n}$ into the two-dimensional domain $\mathcal{B}$ as $\vec{n}(u, \tau)$ and calculating (四).

In terms of $z$-variable we have for Berry phase

$$
W_{E}[\vec{n}]=-i(2 S) \int_{0}^{\beta} d \tau z^{\dagger} i \partial_{\tau} z
$$

which is apparently independent from the auxiliary coordinate $u$. This "simplification" is, however, deceptive because the equation $\vec{n}=z^{\dagger} \vec{\sigma} z$ does not define $z$ unambiguously. One can always change $z(\tau) \rightarrow e^{i \alpha(\tau)} z(\tau)$ with any function $\alpha(\tau)$ satisfying $e^{i \alpha(\beta)}=e^{i \alpha(0)}$. The latter is necessary to conserve periodic boundary conditions $z(\beta)=z(0)$. This "gauge" transformation obviously does not change the vector $\vec{n}$. The change of WZNW action (6) under this transformation is $\delta W_{E}=$ $i(2 S)[\alpha(\beta)-\alpha(0)]=i(2 S) 2 \pi k$ where $k$ is any integer. We arrive to the same result: the action $W_{E}$ itself is multivalued while $e^{-W_{E}}$ is single-valued under the condition that $2 S$ is an integer number!

Let us now briefly discuss topological aspects of Berry phase (WZNW term) for a single spin (雨). The classical unit vector $\vec{n}$ from (4) takes values on a unit twodimensional sphere $S^{2}$ - the target space of the problem. There exists nontrivial expression (the integrand of (4)) depending on vector $\vec{n}$ and its derivatives which is (i) local (ii) being integrated over two-dimensional sphere gives integer values for all possible smooth configurations of $\vec{n}$ defined on $S^{2}$. Mathematically we say that there exists a closed but non-exact 2-form on $S^{2}$ (target space) or even more formally that the second cohomology group of $S^{2}$ is $H_{2}\left(S^{2}\right)=Z$. This allows one to write down the topological term (14) where an integration of the mentioned 2 -form is performed over a domain $\mathcal{B}$ with physical time as the boundary of this disk. The special properties mentioned above guarantee that (4) unambiguously defines the path integral (3).

\section{DOUBLE EXCHANGE MODEL IN THE LIMIT OF INFINITE $J_{H}$}

Let us now consider the double exchange model (1) and take a limit of infinite Hund's constant $J_{H} / t \rightarrow \infty$. We will do it in the Lagrangian formulation. First we use the WZNW action (6) to write the Lagrangian (in this section we use real time representation) of the double exchange model corresponding to the Hamiltonian (1D)

$$
L=\sum_{i}\left[c_{i}^{\dagger}\left(i \partial_{t}-\mu\right) c_{i}+2 S z_{i}^{\dagger} i \partial_{t} z_{i}\right]-H .
$$

Here $\mu$ is a chemical potential which is chosen to guarantee the correct filling factor of electrons and $z_{i}=$ $\left(z_{i 1}, z_{i 2}\right)^{t}$ is a complex vector corresponding to the core spin $S_{i}$ at the site $i$ of the lattice. We will make the following change of variables in the Lagrangian $c_{i}=$ $\psi_{i} z_{i}+\tilde{\psi}_{i} \tilde{z}_{i}$. Here $\psi_{i}, \tilde{\psi}_{i}$ are spinless fermions, $z_{i}$ is a core spin variable, and $\tilde{z}_{i}$ is a complex vector orthogonal to $z_{i}$, namely, $\tilde{z}_{i}=\left(z_{i 2}^{*},-z_{i 1}^{*}\right)^{t}$. Physically, $\psi_{i}$ is a component of an electron on the site $i$ with a spin parallel to the core spin at the same site and $\tilde{\psi}_{i}$ is the one with a spin antiparallel to the core spin. Taking limit $J_{H} \rightarrow \infty$ corresponds to neglecting $\tilde{\psi}$ component of an electron. We substitute $c_{i}=\psi_{i} z_{i}$ into (7) and omitting constant terms obtain

$$
\begin{aligned}
L & =\sum_{i} \psi_{i}^{\dagger}\left(i \partial_{t}-\mu\right) \psi_{i}+\sum_{\langle i j\rangle}\left[\psi_{i}^{\dagger}\left(t z_{i}^{\dagger} z_{j}\right) \psi_{j}+\text { h.c. }\right] \\
& +\sum_{i}\left(2 S+\psi_{i}^{\dagger} \psi_{i}\right) z_{i}^{\dagger} i \partial_{t} z_{i} .
\end{aligned}
$$

The first term of (8) is a kinetic term for projected electrons. Let us take a close look at the second and the third terms. We assume that the motion of spins is much slower then the motion of electrons. This is justified in the limit of large core spins $S \gg 1$. Then one can think of electrons as moving in the background of almost static $z$ 's. The second term in (8) describes the hopping of these electrons with the effective hopping amplitude $t_{i j}^{e f f}=$ $t z_{i}^{\dagger} z_{j}$. This amplitude is complex number and has both the magnitude and the phase.

The magnitude of the effective hopping amplitude is given by $\left|t_{i j}^{e f f}\right|^{2} \equiv \Delta^{2}=t^{2}\left(z_{i}^{\dagger} z_{j}\right)\left(z_{j}^{\dagger} z_{i}\right)=t^{2} \frac{1+\vec{n}_{i} \vec{n}_{j}}{2}$ or $\Delta=t \cos \frac{\theta_{i j}}{2}$ where $\theta_{i j}$ is the angle between two core spins. In this derivation we used a relation $\vec{n}_{i}=z_{i}^{\dagger} \vec{\sigma} z_{i}$ and the known identity on Pauli matrices $\vec{\sigma}_{\alpha \beta} \vec{\sigma}_{\gamma \delta}=$ 
$2 \delta_{\alpha \delta} \delta_{\beta \gamma}-\delta_{\alpha \beta} \delta_{\gamma \delta}$. The absolute value of the effective hopping is proportional to the cosine of the half of the angle between core spins [2]. It is maximal and equal $t$ for parallel core spins and vanishes for antiparallel spins.

The phase of the effective amplitude $t_{i j}^{e f f}$ is not defined unambiguously because of the gauge freedom (see the previous section). Consider, however, an electron moving along some closed trajectory in a background of a smooth configuration of core spins. Then along the path we can write $z_{i+1}=z_{i}+\delta z_{i}$, where $\delta z_{i}$ is small, and $z_{i}^{\dagger} z_{i+1}=1+z_{i}^{\dagger} \delta z_{i}$. The product of all the effective amplitudes along the path is $\prod_{i} t_{i, i+1}^{e f f} \sim \exp \left(\sum_{i} \ln z_{i}^{\dagger} z_{i+1}\right) \approx$ $\exp \left(\sum_{i} z_{i}^{\dagger} \delta z_{i}\right)=\exp i \sum_{i}\left(\delta \psi_{i}+\delta \phi_{i} \sin ^{2} \theta_{i} / 2\right)$, where we use parameterization $z_{i}^{t}=e^{i \psi_{i}}\left(\cos \theta_{i} / 2, e^{i \phi_{i}} \sin \theta_{i} / 2\right)$ of $z_{i}$ in terms of Euler angles $\theta_{i}, \phi_{i}$, and $\psi_{i}$. Along the closed path $\sum_{i} \delta \psi_{i}=0$, so only the second term contributes to the phase picked up by an electron. It is easy to see that this second term is equal to the half of the solid angle enclosed by vectors $\vec{n}$ along the trajectory of an electron. We see that (projected) electrons moving along some closed trajectory acquire the phase equal to the half of solid angle formed by spins along this trajectory. One can say, therefore, that they "feel" the solid angle formed by core spins as a flux of magnetic field. This flux is (modulo $2 \pi$ ) a gauge invariant and well defined quantity.

Finally, the last term in (8) is a "modified" kinetic term for core spins. One might expect that after the averaging over electrons this term will describe the dynamics of effective spins of magnitude $S+\left\langle\psi^{\dagger} \psi\right\rangle / 2$. However, having non-integer coefficient in front of Berry phase results in an inconsistent theory.

To make this seeming paradox more precise we average over electrons accurately by integrating fermions [10] out of (8). We obtain an effective action for core spins in terms of $z$-variables

$$
\begin{aligned}
W & =-i \ln \operatorname{det}\left[\delta_{i j}\left(i \partial_{t}-\mu+z_{i}^{\dagger} i \partial_{t} z_{i}\right)+t z_{i}^{\dagger} z_{j}\right] \\
& +\int d t \sum_{i} 2 S z_{i}^{\dagger} i \partial_{t} z_{i} .
\end{aligned}
$$

Here the expression in the determinant is both an operator in time and $N \times N$ matrix in lattice sites. The matrix element $z_{i}^{\dagger} z_{j}$ is present only if $i, j$ are nearest neighbors. In the adiabatic limit there are two main effects produced by an integration over fermionic degrees of freedom. First, the energy of the filled (to the filling factor $x$ ) Fermi sea is added to the action. This leads to an effective ferromagnetic interaction between core spins. Second, the correction to the Berry phase of core spins must reflect the fact that spins of conduction electrons are effectively added to core spins. The latter correction is the matter of consideration of this paper.

The variation of (9) over $z_{i}^{\dagger} i \partial_{t} z_{i}$ immediately gives $\left(2 S+\left\langle\psi_{i}^{\dagger} \psi_{i}\right\rangle\right)$, where $\left\langle\psi_{i}^{\dagger} \psi_{i}\right\rangle$ are some fractional numbers which in general depend on the configuration of core spins $\left(z_{i}\right.$ 's). This implies the presence of the term $\left(2 S+\left\langle\psi_{i}^{\dagger} \psi_{i}\right\rangle\right) z_{i}^{\dagger} i \partial_{t} z_{i}$ in the action (9) and confirms the result we expected from the heuristic "averaging" over electrons. However, the presence of this term alone renders theory inconsistent.

In the next section we calculate the fermionic determinant of the type (9) albeit for a simplified double exchange model on two lattice sites. We show that there is an additional term present in the effective action for core spins. This additional term will make the effective theory consistent as it is shown in section $\mathrm{V}$.

\section{DOUBLE EXCHANGE MODEL FOR TWO CORE SPINS}

The paradox of having fractional spin in the effective action for a double exchange model is present already for a much simpler double exchange model on two lattice sites with a single conduction electron. In addition the electron spectrum in such model is discrete which makes integration over fermions in adiabatic approximation to be a well-defined procedure. Therefore, in this section we consider the double exchange model on two lattice sites with one conduction electron and show how to resolve the issue of fractional spin on this simple example.

The projected $\left(J_{H} \rightarrow \infty\right)$ double exchange model (8) on two lattice sites (in imaginary time)

$$
\begin{aligned}
L_{E} & =\sum_{i=1,2}\left[\psi_{i}^{\dagger}\left(\partial_{\tau}+i \mu\right) \psi_{i}+\left(2 S+\psi_{i}^{\dagger} \psi_{i}\right) z_{i}^{\dagger} \partial_{\tau} z_{i}\right] \\
& -t\left[\psi_{1}^{\dagger}\left(z_{1}^{\dagger} z_{2}\right) \psi_{2}+\text { h.c. }\right],
\end{aligned}
$$

where $W_{E}=\int_{0}^{\beta} d \tau L_{E}$ is a Euclidean action. Chemical potential $\mu$ must be chosen to guarantee that there is exactly one conduction electron in the system, i.e., $\sum_{i=1,2} \psi_{i}^{\dagger} \psi_{i}=1$. One can repeat the heuristic argument of the previous section replacing $\psi_{i}^{\dagger} \psi_{i}$ in the second term of (10) by its average value $1 / 2$. One obtains two effective spins of magnitude $S+1 / 4$ instead of bare core spins. Again, we have the problem of having the fractional coefficient in the Berry phase term.

We rewrite the Lagrangian (10) as

$$
L_{\mathrm{E}}=\sum_{i=1,2}\left[2 S z_{i}^{\dagger} \partial_{\tau} z_{i}\right]+\Psi^{\dagger}\left(\partial_{\tau}+i \mu+\hat{A}\right) \Psi .
$$

Here we defined $\Psi^{\dagger}=\left(\psi_{1}^{\dagger}, \psi_{2}^{\dagger}\right)$ and $2 \times 2$ matrix $\hat{A}$

$$
\hat{A}=\left(\begin{array}{cc}
z_{1}^{\dagger} \partial_{\tau} z_{1} & -\Delta e^{-i a} \\
-\Delta e^{i a} & z_{2}^{\dagger} \partial_{\tau} z_{2}
\end{array}\right),
$$

where we introduced explicitly the phase and the magnitude of an effective hopping amplitude

$$
t z_{1}^{\dagger} z_{2}=\Delta e^{-i a} .
$$

The absolute value of an effective hopping amplitude $\Delta=t \cos \theta / 2$, where $\theta$ is an angle between core spins 
$\vec{S}_{1}$ and $\vec{S}_{2}$. It is equal to zero only for strictly antiparallel core spins $\theta=\pi$. For fixed directions of core spins the matrix $\hat{A}$ becomes constant matrix with eigenvalues $\pm \Delta$ corresponding to the two-level spectrum of an electron hopping between two core sites in double exchange model with infinite $J_{H}$. Two-level spectrum is symmetric and one can choose the chemical potential $\mu=0$ so that there is only one filled energy level at any given moment of time. Then the spinless fermion $\psi$ (electron with projected spin) occupies the lowest energy level $E=-\Delta$ which is minimal $\left(E_{\min }=-t\right)$ when core spins are parallel. Changing the angle between core spins requires the energy of the order of $t$ while we are interested in low energy dynamics with typical values of energy $\sim t / S$. Therefore, we assume that $\Delta$ does not vanish and is of the order of $t$.

The Grassman variable $c(\tau)$ corresponding to the conduction electron must satisfy antiperiodic boundary conditions in imaginary time $\tau: c_{i}(\beta)=-c(0)$. We represent $c_{i}(\tau)=\psi_{i}(\tau) z_{i}(\tau)$ as a product of spinless fermion $\psi_{i}(\tau)$ and spin $1 / 2$ boson $z_{i}(\tau)$, which after projection $J_{H} \rightarrow \infty$ becomes $z$-boson corresponding to core spins. The boundary conditions for $\psi_{i}(\beta)=-\psi_{i}(0)$ and $z_{i}(\beta)=z_{i}(0)$ are antiperiodic and periodic respectively. Then we have for $\Psi(\tau)$ and $a(\tau)$ (see (11, 13))

$$
\begin{aligned}
\Psi(\beta) & =-\Psi(0), \\
a(\beta) & =a(0)+2 \pi k,
\end{aligned}
$$

where $k$ is an integer - the winding number of the phase $a$ in imaginary time $2 \pi k=\int_{0}^{\beta} d \tau \partial_{\tau} a$.

The effective action of core spins is given by

$$
W_{E}^{e f f}=\int_{0}^{\beta} \sum_{i=1,2}\left[2 S z_{i}^{\dagger} \partial_{\tau} z_{i}\right] d \tau+W_{E}^{i n d},
$$

where the effective action of core spins induced by fermions

$$
W_{E}^{i n d}=-\operatorname{Tr} \ln \left(\partial_{\tau}+\hat{A}\right) .
$$

Here the operator $\partial_{\tau}+\hat{A}$ is linear differential operator with explicit dependence on time $\tau$ through the $2 \times 2$ matrix $\hat{A}$ and we put $\mu=0$ corresponding to having only one conduction electron in the system. To calculate the functional trace (17) we find the eigenvalues of this operator by solving differential equation

$$
\left(\partial_{\tau}+\hat{A}\right) \Psi(\tau)=\lambda \Psi(\tau)
$$

with boundary conditions (14, 15) and calculate the sum of logarithms of all eigenvalues $\lambda$. Matrix (12) can be rewritten as

$$
\hat{A}=\frac{i}{2} A_{0}-e^{-\frac{i}{2} a \sigma^{3}} \Delta \sigma^{1} e^{\frac{i}{2} a \sigma^{3}}+i a_{0} \sigma^{3},
$$

where

$$
\begin{aligned}
i A_{0} & =z_{1}^{\dagger} \partial_{\tau} z_{1}+z_{2}^{\dagger} \partial_{\tau} z_{2} \\
i a_{0} & =\frac{1}{2}\left(z_{1}^{\dagger} \partial_{\tau} z_{1}-z_{2}^{\dagger} \partial_{\tau} z_{2}\right)
\end{aligned}
$$

We would like to solve (18) with the matrix $\hat{A}$ from (19) in an adiabatic approximation, e.g., assuming that all time derivatives are much smaller than the energy scale $\Delta \sim t$. First we "unwind" wavefunction $\Psi$ by a unitary transformation

$$
\Psi=\exp \left\{-\frac{i}{2} \int_{0}^{\tau} d \tau\left(A_{0}+\dot{a} \sigma^{3}\right)\right\} \chi,
$$

where $\dot{a}$ means the derivative of $a$ over $\tau$. Then, instead of (18) the function $\chi$ satisfies

$$
\left(\hat{L}_{0}+\hat{V}\right) \chi=\lambda \chi
$$

where we defined "zero order" operator $\hat{L}_{0}$ and perturbation $\hat{V}$ which is proportional to time derivatives of core spins as

$$
\begin{aligned}
\hat{L}_{0} & =\partial_{\tau}-\Delta \sigma^{1}, \\
\hat{V} & =i \tilde{a}_{0} \sigma^{3}, \\
\tilde{a}_{0} & =a_{0}-\frac{1}{2} \dot{a} .
\end{aligned}
$$

Now we can solve (21) using the perturbation theory in $\hat{V}$. The boundary conditions of $\chi$ are given by (14) and (20) as

$$
\chi(\beta)=-e^{i \gamma} \chi(0),
$$

where Berry phase

$$
\gamma=\frac{1}{2} \int_{0}^{\beta}\left(A_{0}+\dot{a}\right) d \tau,
$$

where we used that $\int_{0}^{\beta} \dot{a} d \tau=2 \pi k$ ( $k$ is an integer, see (15)) and that $e^{i \pi k \sigma^{3}}=e^{i \pi k}$.

We are going to find the eigenvalues of (21) using the perturbation theory in $\hat{V}$. This is justified in an adiabatic limit when $\tilde{a}_{0}$ proportional to the rate of change of core spins is much smaller than the typical fermionic energy $\Delta$ or in other words when the conduction electron is the fast degree of freedom compared to core spins.

The solutions of the zero order equation

$$
\hat{L}_{0} \chi^{(0)}=\lambda^{(0)} \chi^{(0)}
$$

are labelled by an integer number $n$ and by plus or minus sign

$$
\left|\chi_{n \pm}^{(0)}\right\rangle=\frac{1}{\sqrt{2 \beta}} e^{\mp \int_{0}^{\tau} \Delta d \tau+\lambda_{n \pm}^{(0)} \tau}\left(\begin{array}{c}
1 \\
\pm 1
\end{array}\right)
$$

with eigenvalues

$$
\lambda_{n \pm}^{(0)}= \pm \bar{\Delta}+\frac{i \pi}{\beta}(2 n+1)+\frac{i}{\beta} \gamma
$$

where

$$
\bar{\Delta}=\frac{1}{\beta} \int_{0}^{\beta} \Delta d \tau
$$


There are no corrections to these eigenvalues in the first order of perturbation theory in $\hat{V}$. Therefore, up to the second order of perturbation theory we obtain

$$
\lambda_{n \pm}=-\mu \pm \bar{\Delta}+\frac{i \pi}{\beta}\left(2 n+1+\frac{\gamma}{\pi}\right),
$$

where we have restored the chemical potential $\mu$ which should be $\mu=0$ for the problem with a single conduction electron.

The effective action (17) is given by

$$
W_{E}^{i n d}=-\sum_{n=-\infty}^{+\infty}\left[\ln \lambda_{n+}+\ln \lambda_{n-}\right]
$$

To calculate the obviously divergent sum in (24) we regu- larize it by subtracting the effective action for the system without conduction electrons. That is we calculate the difference between (24) calculated at $\mu=0$ and the one calculated at $\mu \rightarrow-\infty$.

$$
\begin{aligned}
W_{E}^{i n d} & =-\sum_{n=-\infty}^{+\infty}\left[\ln \frac{\lambda_{n+}(\mu=0)}{\lambda_{n+}(\mu \rightarrow-\infty)}\right. \\
& \left.+\ln \frac{\lambda_{n-}(\mu=0)}{\lambda_{n-}(\mu \rightarrow-\infty)}\right] .
\end{aligned}
$$

This sum is still logarithmically divergent but one can show that the divergent part is a constant not depending on physical parameters $\Delta$ and $\gamma$. Calculating the sum in (25) we obtain

$$
W_{i n d}=-\lim _{\mu \rightarrow-\infty} \ln \frac{\cosh \left(\frac{\beta \bar{\Delta}}{2}+i \frac{\gamma}{2}\right) \cosh \left(\frac{\beta \bar{\Delta}}{2}-i \frac{\gamma}{2}\right)}{\cosh \left(\frac{\beta(\bar{\Delta}-\mu)}{2}+i \frac{\gamma}{2}\right) \cosh \left(\frac{\beta(\bar{\Delta}+\mu)}{2}-i \frac{\gamma}{2}\right)}
$$

In the limit $\mu \rightarrow-\infty$ we obtain

$$
\begin{aligned}
W_{i n d} & =i \gamma-\ln \cosh \left(\frac{\beta \bar{\Delta}}{2}-i \frac{\gamma}{2}\right) \\
& -\ln \cosh \left(\frac{\beta \bar{\Delta}}{2}+i \frac{\gamma}{2}\right) .
\end{aligned}
$$

This is the final result for the effective action calculated at finite "temperature" $\beta$. Notice that the only imaginary part of a Euclidean effective action is the Berry phase $\gamma$. We have obtained this phase in the first order of perturbation theory. However, the same expression is also valid in all orders of perturbation theory. It is easy to show for our simple model because the higher orders of perturbation theory can only give corrections to the real part of (23) which does not contribute to the imaginary part of an Euclidean action. We consider the higher orders of perturbation theory using the regular gradient expansion in Appendix B. In fact, the imaginary part of a Euclidean action has a topological nature and, therefore, can not be changed by small perturbations (see the discussion in section (V).

In the "zero temperature" limit $\beta \Delta \gg 1$ we have

$$
W_{E}^{i n d}=i \gamma-\beta \bar{\Delta}
$$

or explicitly

$$
W_{E}^{\text {ind }}=\frac{i}{2} \int_{0}^{\beta}\left(A_{0}+\dot{a}\right) d \tau-\int_{0}^{\beta} \Delta d \tau .
$$

The latter result can of course be obtained just by replacing the discrete sum over $n$ in (25) by an integral.
For the full effective action (16) we obtain

$$
\begin{aligned}
W_{E}^{e f f} & =W_{E}^{B}+W_{E}^{d y n} \\
W_{E}^{B} & =\int_{0}^{\beta} d \tau\left[\sum_{i=1,2}\left(2 S+\frac{1}{2}\right) z_{i}^{\dagger} \partial_{\tau} z_{i}+\frac{i}{2} \dot{a}\right] \\
W_{E}^{d y n} & =-\int_{0}^{\beta} \Delta d \tau+\ldots
\end{aligned}
$$

The first term of the dynamic part of an effective action $W_{E}^{d y n}$ is just the time integral of the energy of the ground state of the conduction electron in a fixed instantaneous configuration of core spins. The dots denote the higher order corrections to (31). These corrections are small if the background core spins are moving adiabatically and they are diverging if derivatives $\dot{\vec{n}}_{1}, \vec{n}_{2}$ are bigger than $\Delta$. For such processes an adiabatic approximation fails. In particular, if core spins have opposite directions $\vec{n}_{1}=$ $-\vec{n}_{2}$ we have $\Delta=0$ and an adiabatic approximation is not applicable at any rate of the change of core spins.

\section{BERRY PHASE FOR FRACTIONAL SPINS}

\section{A. Consistency}

The Berry phase term (30) is the main result of this paper. We see that the the correction to the Berry phase of bare core spins induced by the conduction electron is twofold. First, as we expected averaging over fermions in (10) there is a change of the coefficient in Berry phases 
of core spins from $2 S$ to $2 S+1 / 2$. In addition, there is a correction $\frac{i}{2} \int_{0}^{\beta} d \tau \dot{a}=i \pi k$, where the integer number $k$ is a winding number (15) of the phase $a$ in imaginary time. The latter term is a full time derivative and, therefore, does not change the classical dynamics of spins. Classically, the effective "core" spins behave as if they had the value of $S+1 / 4$ !

Let us show now that the full Berry phase (30) is consistent with quantum mechanics of spins and, therefore, answers the question of how to write down the Berry phase for two fractional spins $S+1 / 4$. As we have seen at the end of the section [1] the inconsistency occurs if the partition function is not invariant under gauge transformations of $z$ variables. We perform the gauge transformation $z_{1,2} \rightarrow e^{i \alpha_{1,2}(\tau)} z_{1,2}$ independently for first and second core spins. In order not to change the periodic boundary conditions of $z_{1,2}$ we require $\Delta \alpha_{1,2}=\alpha_{1,2}(\beta)-\alpha_{1,2}(0)=2 \pi l_{1,2}$, where $l_{1,2}$ are arbitrary integer numbers. Under this transformation the three terms of (30) depending on $z_{1}, z_{2}$, and $a$ change by $2 \pi i l_{1}(2 S+1 / 2), 2 \pi i l_{2}(2 S+1 / 2)$, and $\pi i\left(l_{1}-l_{2}\right)$ respectively. It is easy to see that neither of these three terms gives an invariant contribution to a partition function. E.g., for $l_{1}=1$ the change of the first term is $2 \pi i(2 S+1 / 2)$ which gives -1 being exponentiated. However, the total kinetic term (30) is equal to the sum of all these terms and changes under the gauge transformation by $2 \pi i\left[(2 S+1) l_{1}+2 S l_{2}\right]$ which is a multiple of $2 \pi$ and, therefore, does not change the weight $e^{-W_{E}^{B}}$. This means that neither of three terms considered makes any sense separately from the others 114. Only their gauge invariant (modulo $2 \pi$ ) sum has a physical meaning of the combined Berry phase of the system of two core spins plus one conduction electron.

\section{B. Topology and adiabatic approximation}

We have seen in section II that the existence of WZNW term for a single unit vector (single spin) is due to the fact that the target space $S^{2}$ has a nontrivial second cohomology group $H_{2}\left(S^{2}\right)=Z$. The addition of WZNW term to the action with the coefficient $2 S$ is consistent only if this coefficient is quantized (is an integer number). For two independent spins the target space becomes $S^{2} \times S^{2}$ and one obviously can write two independent WZNW terms corresponding to $H_{2}\left(S^{2} \times S^{2}\right)=Z \times Z$. This is apparently true for two independent spins. However, the result is different for the conduction electron induced dynamics of core spins in the double exchange model. Let us discuss why this is so. To obtain the induced dynamics of core spins we have excluded the charge degree of freedom of conduction electron integrating it out in an adiabatic approximation. This approximation, however, breaks when the the rate of the change of core spins becomes comparable to the energy scale $2 \Delta$ given by the levels of conduction electron in the background of time independent core spins. There is a special point though
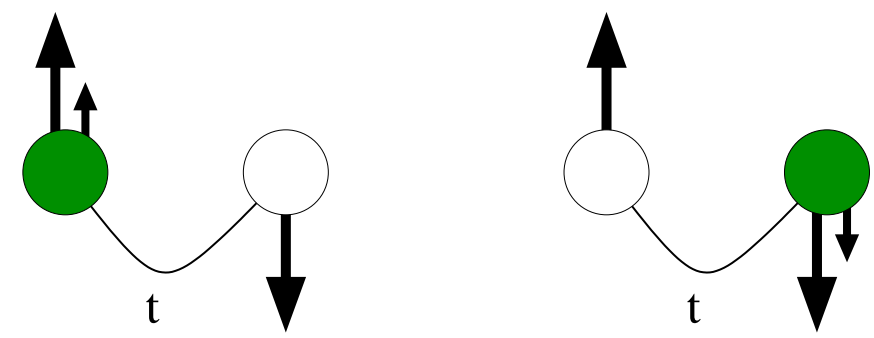

FIG. 2: Two degenerate states with antiparallel core spins. An electron with spin aligned along the core spin can not hop from one lattice site to another and can be either on the right or on the left lattice site.

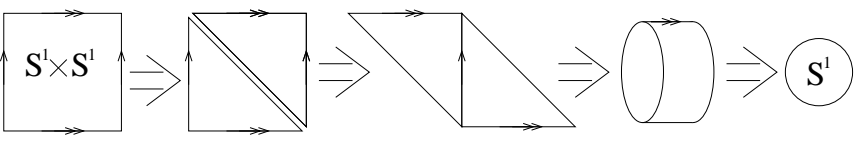

FIG. 3: The contraction $S^{1} \times S^{1} \backslash S^{1} \rightarrow S^{1}$ is shown as a onedimensional illustration of $G \sim S^{2}$. a) The torus space $S^{1} \times S^{1}$ is represented as a square with the opposite sides identified according to the direction of arrows. The diagonal shows the points given by $\vec{n}_{1}=-\vec{n}_{2}$. b) The space $S^{1} \times S^{1} \backslash S^{1} \rightarrow S^{1}$ is obtained by the removal of the diagonal. c) One pair of opposite sides is glued. d) The second pair is glued making a cylinder. e) The cylinder is contracted onto the unit circle.

when core spins are opposite to each other. At this point $\Delta=0$ and an adiabatic approximation breaks no matter how slow the motion of core spins is.

This is quite obvious from the physical point of view. Consider two time independent core spins opposite to each other. The effective hopping amplitude of a conduction electron is $\sim \cos \theta / 2=0$ and one has two different configurations. The electron can be either on the left lattice site or on the right one (see Figure 2) and the matrix element of the hopping term is zero between those states. This means that the ground state of the system is doubly degenerate. The charge degree of freedom (left or right position of the electron) is essential for this particular configuration of core spins and can not be integrated out. In our derivation we used an adiabatic approximation and neglected such configurations. This is justified in the limit $S \gg 1$ for ferromagnetically interacting core spins. However, this approximation drastically changes the topology of the system. Essentially, we prohibited the configurations $\vec{n}_{1}=-\vec{n}_{2}$. The relevant target space changes from $S^{2} \times S^{2}$ for two independent spins to $G=S^{2} \times S^{2} \backslash S^{2}$. The latter expression denotes the direct product $S^{2} \times S^{2}$ with points $\vec{n}_{1}=-\vec{n}_{2}$ (topologically equivalent to one more $S^{2}$ ) removed. The resulting target space $G$ is topologically equivalent to $S^{2}$. To prove it one can show 15 that it can be contracted along itself onto $S^{2}$. We illustrate the similar phenomenon on the corresponding one-dimensional example in Figure 3. Therefore, the relevant target space $G \sim S^{2}$. To check for allowed WZNW terms we calculate $H_{2}(G)=H_{2}\left(S^{2}\right)=Z$. There is only one WZNW term 
allowed for the chosen target space!

The existence of only one allowed topological term explains why only the full Berry phase (30) is well defined. The most general form (in $C P^{1}$ representation) of the topological term for two spins is given by

$$
W_{E}^{t o p}=\int_{0}^{\beta} d \tau\left[\rho_{1} z_{1}^{\dagger} \partial_{\tau} z_{1}+\rho_{2} z_{2}^{\dagger} \partial_{\tau} z_{2}+i \xi \dot{a}\right]
$$

Coefficients $\rho_{1,2}, \xi$ satisfy quantization conditions (or gauge invariance conditions). Namely, $\rho_{1}-\xi$ and $\rho_{2}+\xi$ must be integers. We see that for the double exchange model of two core spins and a single conduction electron we have (30) which corresponds to particular integer values $\rho_{1}-\xi=2 S$ and $\rho_{2}+\xi=2 S+1$. If by some reason the conduction electron spends unequal time at two sites (e.g., the local magnetic field acting on electron spin on one of sites is added) we have an asymmetric situation $\rho_{1} \neq \rho_{2}$. However, the combination $\rho_{1}-\xi$ and $\rho_{2}+\xi$ must stay integer to guarantee the consistency of the theory.

\section{CONCLUSION}

In this paper we considered the infinite Hund's coupling limit of the double exchange model with a given concentration $x$ of conduction electrons. In the limit of large core spins $S \gg 1$ one can think of electrons and core spins as of fast and slow degrees of freedom respectively. The superficial averaging over the fast motion of electrons leads to an inconsistent theory with effective fractional spins. Using the example of the double exchange model on two lattice sites we showed that in addition to the change of the value of the effective core spin on site from $S$ to $S+x / 2$ a new topological term arises in the effective action. With this term the exponent of the effective action becomes single-valued and well defined. We showed that the full Berry phase (30) reflects the change of topology of the system which occurs due to the adiabaticity condition - the condition justifying the averaging over the charge degrees of freedom. The generalization of our results to the double exchange model for many spins is relatively straightforward. The only major difference with the case of two spins is that the average occupation number of a given lattice site by conduction electrons depends on the configuration of core spins. This makes the magnitude of a "fractional" spin on the given site a fluctuating quantity with correspondent modifications of Berry phase terms. The latter effect is not expected to be strong in the ferromagnetically ordered state.

\section{Acknowledgments}

We are grateful to Boris Spivak who stimulated us to think about fractional spins in ferromagnetic metals. The discussion of topological aspects of Berry phase with
Dmitri Ivanov is deeply appreciated. A. G. A. is Alfred P. Sloan Research Fellow. The work of Ar. A. is supported by DR Project 200153 at Los Alamos National Laboratory.

\section{APPENDIX A: PATH INTEGRAL FOR SINGLE SPIN AND FERMIONIC DETERMINANT}

In this section for the sake of completeness we rederive the well-known path integral representation for the quantum mechanics of a single spin. We reduce the problem to a calculation of a simple fermionic determinant. The method we use differs from the commonly used coherent state representation (see e.g., [7]) for the path integral. It is conceptually simpler but involves the gradient expansion calculation of a fermionic determinant. It turns out that the similar determinant is needed for our analysis of a double exchange model for two core spins.

\section{Path integral representation for spin $S$}

It is easy to check that the representation $\vec{S}=$ $\frac{1}{2} \psi_{\alpha}^{\dagger} \vec{\sigma}_{\alpha \beta} \psi_{\beta}$ with constraint $\psi_{\alpha}^{\dagger} \psi_{\alpha}=1$ is a faithful representation of (2) with $S=1 / 2$. Here $\vec{\sigma}=\left(\sigma^{x}, \sigma^{y}, \sigma^{z}\right)$ is a set of Pauli matrices and $\psi_{\alpha}, \psi_{\beta}^{\dagger}$ with $\alpha, \beta=1,2$ are annihilation (creation) Fermi operators respectively which satisfy the following (anti)commutation relations $\left\{\psi_{\alpha}, \psi_{\beta}\right\}=\left\{\psi_{\alpha}^{\dagger}, \psi_{\beta}^{\dagger}\right\}=0$ and $\left\{\psi_{\alpha}, \psi_{\beta}^{\dagger}\right\}=\delta_{\alpha \beta}$. In fact, this representation reflects how spin operators appear in Nature with $\psi$ being an annihilation operator of the physical electron.

We consider the following Hamiltonian $H=-2 \Delta \vec{n} \vec{S}$, where $\vec{S}=\frac{1}{2} \psi^{\dagger} \vec{\sigma} \psi$ is a spin of a fermion and $\Delta$ is a strength of the coupling of this spin with the unit vector $\vec{n}$. The correspondent action is

$$
S=\int d t \psi^{\dagger}\left[i \partial_{t}+\Delta \hat{n}\right] \psi
$$

where we introduced a notation $\hat{n}=\vec{n} \vec{\sigma}$. One can think of $2 \Delta \vec{n}$ as of the magnetic field acting on the fermionic spin $\vec{S}$. In the limit of an infinite coupling $\Delta \rightarrow \infty$ (infinite magnetic field) the fermionic spin $\vec{S}$ will be "frozen" along the classical vector $\vec{n}$ so that $\vec{n}$ in the limit of infinite $\Delta$ becomes a direction of quantization axis of a quantum spin of a fermion. If now we change the direction of the classical vector $\vec{n}$ with time, the fermionic spin will follow the direction of $\vec{n}$. Therefore, we expect that in the limit of an infinite $\Delta$ an effective dynamics of classical vector $\vec{n}$ induced by a fermion will be that of the quantum spin $1 / 2$.

The path integral representation for spin $1 / 2$ is then given by

$$
Z=\int D \vec{n} e^{i W[\vec{n}]}
$$


where $W[\vec{n}]=\lim _{\Delta \rightarrow \infty} W^{e f f}[\vec{n}]$ is a classical action corresponding to a quantum spin $1 / 2$. This classical action is a functional of the trajectory of the time dependent unit vector $\vec{n}(t)$. The effective action $W_{\text {eff }}[\vec{n}]$ is defined as

$$
e^{i W^{e f f}[\vec{n}]}=\int D \psi D \psi^{\dagger} e^{i \int d t \psi^{\dagger}\left[i \partial_{t}+\Delta \hat{n}\right] \psi} .
$$

The functional integration in (A3) is performed over Grassman variables 10 $\psi, \psi^{\dagger}-$ classical analogs of fermionic annihilation (creation) operators. Integrating over fermions we obtain

$$
W^{e f f}[\vec{n}]=-i \ln \operatorname{Det}\left[i \partial_{t}+\Delta \hat{n}\right] .
$$

To generalize the result to an arbitrary spin $S$ we can consider the same fermionic theory (A3) but with $2 S$ independent species of fermions coupled to the same vector $\vec{n}$. In this case all spins $1 / 2$ of fermions will be aligned along $\vec{n}$ so that (A2) will correspond to quantum spin $S$. Integration over all fermionic species is independent and we have for an effective action

$$
W^{e f f}[\vec{n}]=-i 2 S \ln \operatorname{Det}\left[i \partial_{t}+\Delta \hat{n}\right] .
$$

The only problem which is left is technical. It is to calculate the fermionic determinant (A5) in the limit of an infinite $\Delta$.

\section{Shortcut}

One can obtain the result without (almost) any calculations. Let us introduce the complex, two-component, normalized vector $z^{t}=\left(z_{1}, z_{2}\right), z^{\dagger} z=1$ so that it is an eigenvector of $\hat{n}$ with the eigenvalue 1 : $\hat{n} z=z$. For a time independent $\hat{n}$ this vector is a ground state of our Hamiltonian $H z=-\Delta \hat{n} z=-\Delta z$ with the ground state energy $-\Delta$. The other eigenstate has an energy $+\Delta$ and in the limit of an infinite $\Delta$ is never excited by a motion of the unit vector $\vec{n}$. Therefore, in the limit of an infinite $\Delta$ we can use $\psi_{\alpha}=z_{\alpha} \chi$ with $\chi$-spinless (projected) fermion. Substituting this into the action (A1) we obtain

$$
S=\int d t\left[\chi^{\dagger}\left(i \partial_{t}+\Delta\right) \chi+\chi^{\dagger} \chi\left(z^{\dagger} i \partial_{t} z\right)\right] .
$$

The variable $\chi$ does not have any dynamics in the limit of an infinite $\Delta$. We average over $\chi$ using $\chi^{\dagger} \chi=1$ and obtain $W=\int d t z^{\dagger} i \partial_{t} z$ for spin $1 / 2$. In case of spin $S$ one has $2 S$ species of fermions, $\chi^{\dagger} \chi=2 S$ and $W=$ $2 S \int d t z^{\dagger} i \partial_{t} z$. This is the desired result for the action for a single spin $S$. However, the main point of this paper is to show that the "naive" averaging over fermions instead of the careful determinant calculation can result in error. In this case one can show that due to topological reasons the calculation we performed is a legitimate one. To add even more weight to this statement we proceed with a formal calculation of the fermionic determinant (A5) and show that it gives the same result for a single spin action.

\section{Determinant calculation}

Before going to the calculation of fermionic determinant A5 we will perform Wick rotation of time and go to an imaginary time representation. Imaginary time representation is slightly more convenient for calculations for reasons that will be clear later. The change to an imaginary time changes quantum amplitude $e^{i W}$ into a "Boltzmann" factor $e^{-W_{E}}$, where subscript "E" stands for "Euclidean". In imaginary time $\tau=i t$ we have instead of A5

$$
W_{E}^{e f f}[\vec{n}]=-2 S \ln \operatorname{Det}\left[\partial_{\tau}-\Delta \hat{n}\right]
$$

with the partition function for a single spin

$$
Z=\int D \vec{n} e^{-W_{E}[\vec{n}]},
$$

where $W_{E}[\vec{n}]=\lim _{\Delta \rightarrow \infty} W_{E}^{\text {eff }}[\vec{n}]$. We impose periodic boundary conditions (in imaginary time) on $\vec{n}(\tau)$ so that $\vec{n}(\beta)=\vec{n}(0)$ and $\beta$ is a maximal span of an imaginary time. With these boundary conditions we can think of time as of the one-dimensional circle with identification of points $\tau=\beta$ with $\tau=0$. Physical results will be obtained in the limit $\beta \rightarrow \infty$ after analytical continuation back to real time $t=-i \tau$.

Using the method presented in Appendix B one can calculate the effective action (A6) in adiabatic approximation with an arbitrary accuracy. However, we are interested in the limit $\Delta \rightarrow \infty$. In this limit all terms of gradient expansion in $1 / \Delta$ except for the first (topological) one will vanish. Here we show how to obtain the most important (topological) $\Delta$-independent part of the effective action (A6) which is the exact result for the action for a single spin.

Determinant of an operator does not change if one applies a unitary transformation to it. This is, however, a potentially dangerous procedure in the context of gradient expansion as one has to make sure the boundary conditions for electrons do not change in the process of such transformation. It turns out that this is not an issue for the single spin problem. However, e.g., for the double exchange problem the boundary condition of fermions do change in a non-trivial way under the unitary rotation (see section IV).

Let us use the transformation $\hat{U}$ such that $\hat{U}^{-1} \hat{n} \hat{U}=$ $\sigma^{3}$. This transformation $\hat{U}$ is easily found using the so called $C P^{1}$ representation. In this representation we introduce a two-component complex vector $z$ with the constraint $z^{\dagger} z=1$ such that $\vec{n}=z^{\dagger} \vec{\sigma} z$. Then the matrix $\hat{U}$ can be written as

$$
\hat{U}=\left(\begin{array}{cc}
z_{1} & z_{2}^{*} \\
z_{2} & -z_{1}^{*}
\end{array}\right) .
$$

We write the equation $\mathrm{A} 6$ ) in the form

$$
W_{E}^{e f f}[\vec{n}]=-2 S \operatorname{Tr} \ln \left[\partial_{\tau}+\hat{A}-\Delta \sigma^{3}\right],
$$


where $\hat{A}=\hat{U}^{-1} \partial_{\tau} \hat{U}$ and $\operatorname{Tr}$ means both functional trace and the trace over $\sigma$-matrices.

The matrix elements of $\hat{A}$ are proportional to the first time derivative of spin variables and are small in comparison to $\Delta$. We can expand the logarithm in (A9) in powers of $\hat{A}$. We keep only the first $\hat{A}$ dependent term as the other terms will have additional powers of $\Delta$ in denominators. (one can obtain those terms in a regular way explained in Appendix B)

$$
\begin{aligned}
W_{E}^{e f f}[\vec{n}] & =2 S \operatorname{Tr} \frac{\partial_{\tau}+\Delta \sigma^{3}}{-\partial_{\tau}^{2}+\Delta^{2}} \hat{A} \\
& =i \int \frac{d \omega}{2 \pi} \operatorname{tr} \frac{-\omega+i \Delta \sigma^{3}}{\omega^{2}+\Delta^{2}} \hat{A}(\omega=0) \\
& =2 S \frac{1}{2} \int_{0}^{\beta} d \tau \operatorname{tr} \sigma^{3} \hat{A} \\
& =-i 2 S \int_{0}^{\beta} d \tau z^{\dagger} i \partial_{\tau} z
\end{aligned}
$$

It is instructive to rewrite $\mathrm{A} 10$ in terms of the original variables $\vec{n}$. We parameterize complex vector $z^{t}=e^{i \psi}\left(\cos \frac{\theta}{2}, e^{i \phi} \sin \frac{\theta}{2}\right)$. It is easy to see that the angles $\theta$ and $\phi$ are the polar and azimuthal angles corresponding to the direction of the unit vector $\vec{n}$. The phase $\psi$ can be chosen arbitrarily as it does not affect $\vec{n}$. Up to the full time derivative of $\psi$ we obtain $-\int_{0}^{\beta} d \tau z^{\dagger} i \partial_{\tau} z=\frac{1}{2} \int_{0}^{\beta} d \tau(1-\cos \theta) \dot{\phi}=\Omega / 2$, where $\Omega$ is a solid angle enclosed by the unit vector $\vec{n}$ during its time evolution. One can check that this solid angle can be rewritten in the form (田) which is explicitly rotationally invariant.

\section{APPENDIX B: CALCULATION OF FERMIONIC DETERMINANT FOR DOUBLE EXCHANGE MODEL}

In this appendix we calculate the effective action (17) which can be written as

$$
W_{E}^{\text {ind }}=-\operatorname{Tr} \ln D,
$$

where the differential operator $D$ is defined as

$$
D=\partial_{\tau}+\hat{A}
$$

with matrix $\hat{A}$ given by (12,19). We rewrite

$$
\hat{A}=i A_{0} / 2+i a_{0} \sigma^{3}+\Delta_{1} \sigma^{1}+\Delta_{2} \sigma^{2}=i A_{0} / 2+\vec{\Delta} \vec{\sigma},
$$

where $\Delta_{1}=-\Delta \cos a, \Delta_{2}=-\Delta \sin a$, and $\Delta_{3}=i a_{0}$.

We use an adiabatic approximation using the (gradient) expansion in $1 / \Delta$. The operator $D$ contains the magnitude of the effective hopping $\Delta$ which is slowly varying but it is not small itself. To avoid this difficulty we calculate instead of effective action its variation and then restore the result from this variation

$$
\delta W_{E}^{i n d}=-\operatorname{Tr} \delta D D^{-1}=-\operatorname{Tr}\left[\delta \hat{A} \frac{1}{\partial_{\tau}+\hat{A}}\right],
$$

where

$$
\delta \hat{A}=i \delta A_{0} / 2+\delta \vec{\Delta} \vec{\sigma} .
$$

Let us now calculate the functional trace in the basis of plane waves

$$
\delta W_{E}^{i n d}=-\operatorname{tr} \int \frac{d \omega}{2 \pi} \int d \tau e^{-i \omega \tau} \delta \hat{A} \frac{1}{\partial_{\tau}+\hat{A}} e^{i \omega \tau}
$$

Here the integral over time $\tau$ is the calculation of the diagonal matrix element $\left\langle\omega\left|\delta D D^{-1}\right| \omega\right\rangle$ and integration over $\omega$ is the calculation of the functional trace. The residual trace $\operatorname{tr}$ is the trace in the space of $2 \times 2$ matrices. "Pulling" $e^{i \omega \tau}$ from right to the left we have

$$
\delta W_{E}^{i n d}=-\operatorname{tr} \int \frac{d \omega}{2 \pi} \int d \tau \delta \hat{A} \frac{1}{i \omega+\partial_{\tau}+\hat{A}} .
$$

In the latter expression the partial derivative $\partial_{\tau}$ is assumed to act "to the right". This means that when we expand

$$
\begin{aligned}
\delta W_{E}^{\text {ind }} & =-\operatorname{tr} \int d t \int \frac{d \omega}{2 \pi} \delta \hat{A} G\left[1-\partial_{\tau} G+\partial_{\tau} G \partial_{\tau} G+\ldots\right] \\
& =\int d t\left[\delta L_{0}+\delta L_{1}+\delta L_{2}+\ldots\right]
\end{aligned}
$$

all derivatives are assumed to act on all terms to the right. E.g., $\partial_{\tau} G \partial_{\tau} G=G \ddot{G}+\dot{G} \dot{G}$. We introduced

$$
G=\frac{1}{i \omega+\hat{A}}=\frac{-i\left(\omega+A_{0} / 2\right)+\vec{\Delta} \vec{\sigma}}{\left(\omega+A_{0} / 2\right)^{2}+|\vec{\Delta}|^{2}}
$$

and

$$
\begin{aligned}
\delta L_{0} & =-\operatorname{tr} \int \frac{d \omega}{2 \pi} \delta \hat{A} G, \\
\delta L_{1} & =\operatorname{tr} \int \frac{d \omega}{2 \pi} \delta \hat{A} G \partial_{\tau} G, \\
\delta L_{2} & =-\operatorname{tr} \int \frac{d \omega}{2 \pi} \delta \hat{A} G \partial_{\tau} G \partial_{\tau} G .
\end{aligned}
$$

We notice that $|\vec{\Delta}|^{2}=\Delta^{2}-a_{0}^{2} \approx \Delta^{2}$ because $a_{0} \ll \Delta$ by adiabatic approximation (it contains time derivative of core spins). Therefore, the denominator of (B4) is never zero and integrals (B5) are not singular. The expansion (B3) is essentially the gradient expansion, i.e., the expansion in the number of time derivatives. One can see that $L_{1}$ contains at least one time derivative, $L_{2}$ - at least two etc.

The calculation of $\delta L_{0}$ is straightforward and gives

$$
\delta L_{0}=\delta\left[i A_{0} / 2-|\vec{\Delta}|\right]
$$

or removing variation

$$
L_{0}=i \frac{A_{0}}{2}-|\vec{\Delta}| \approx i \frac{A_{0}}{2}-\Delta+\frac{a_{0}^{2}}{2 \Delta} .
$$


To obtain the latter expression we expanded up to the second order in time derivatives using $|\vec{\Delta}|=\sqrt{\Delta^{2}-a_{0}^{2}} \approx$ $\Delta-a_{0}^{2} / 2 \Delta$.

Let us now prove that the whole dependence of the full result (B3) on $A_{0}$ comes from $L_{0}$. Varying the action (11) over $A_{0}$ we obtain $\frac{i}{2}\left\langle\Psi^{\dagger} \Psi\right\rangle=\frac{i}{2}$. This statement is exact because the total number of electrons in the system is fixed $\left\langle\Psi^{\dagger} \Psi\right\rangle=1$. For the effective action we have, therefore, $\frac{\delta W_{E}^{i n d}}{\delta A_{0}}=\frac{i}{2}$. However, from (B6) we have $\frac{\delta L_{0}}{\delta A_{0}}=$ $\frac{i}{2}$ which gives $\frac{\delta\left(W_{E}^{i n d}-L_{0}\right)}{\delta A_{0}}=0$ and all higher order terms $L_{1}, L_{2}$ etc. do not depend on $A_{0}$. This simplifies further calculations allowing to use

$$
G=\left.\frac{1}{i \omega+\hat{A}}\right|_{A_{0}=0}=\frac{-i \omega+\vec{\Delta} \vec{\sigma}}{\omega^{2}+|\vec{\Delta}|^{2}}
$$

for all higher order terms.

As a result of calculations one obtains from (B5)

$$
\delta L_{1}=\frac{i}{2} \epsilon^{a b c}\left(\delta \frac{\Delta^{a}}{|\vec{\Delta}|}\right) \frac{\Delta^{b}}{|\vec{\Delta}|} \partial_{\tau} \frac{\Delta^{c}}{|\vec{\Delta}|}
$$

This is identical in form to a Berry phase of a single spin $1 / 2$ given by (5) with identification $\vec{n}=\vec{\Delta} /|\vec{\Delta}|$. Therefore, $L_{1}$ is given by formula analogous to the Berry phase for a single spin (4) and is to be interpreted as a half of solid angle swept by vector $\vec{\Delta}$ during its time evolution. Assume for a moment now that $a_{0}=0$. Then the vector $\vec{\Delta} /|\vec{\Delta}|=(-\cos a,-\sin a, 0)$ moves along the equator of unit sphere and covers the solid angle $\Omega=a(\beta)-a(0)$ during its motion. In this approximation we obtain
$L_{1}=i \dot{a} / 2$ which gives half of the solid angle after time integration 16]. Calculating corrections due to nonzero $a_{0}$ from (B8) we obtain

$$
L_{1}=\frac{i}{2}\left(\dot{a}-\frac{i a_{0} \dot{a}}{|\vec{\Delta}|}\right) \approx \frac{i}{2} \dot{a}-\frac{a_{0} \dot{a}}{2 \Delta},
$$

where we expanded the latter expression up to the terms of the second order in time derivatives.

The calculation of $L_{2}$ in (B5) is straightforward and gives

$$
L_{2}=\frac{1}{8|\vec{\Delta}|}\left(\partial_{\tau} \frac{\vec{\Delta}}{|\vec{\Delta}|}\right)^{2} \approx \frac{\dot{a}^{2}}{8 \Delta}
$$

Finally, combining terms (B7, B9, B10) we obtain an effective action up to the second order terms

$$
L_{E}=\frac{i}{2}\left(A_{0}+\dot{a}\right)-\Delta+\frac{1}{2 \Delta}\left(a_{0}-\frac{\dot{a}}{2}\right)^{2}
$$

It is interesting to notice that although neither of contributions $(\mathrm{B} 7, \mathrm{~B} 9, \mathrm{~B} 10)$ is gauge invariant, the full action (B11) is gauge invariant. This means that the final result (B11) can be rewritten in terms of two unit vectors $\vec{n}_{1}$ and $\vec{n}_{2}$ which represent physical degrees of freedom core spins of double exchange model (11).

The method of gradient expansion used in this appendix can of course also give higher order corrections to $(\mathrm{B} 11)$.
[1] C. Zener, Phys. Rev. 82, 403 (1951).

Interaction between the $d$-shells in the transition metals. 2. Ferromagnetic compounds of manganese with perovskite structure.

[2] P. W. Anderson and H. Hasegawa, Phys. Rev. 100, 675 (1955).

Considerations on double exchange.

[3] The limit of $J_{H} \gg t, S \gg 1$ of double exchange model can be a good starting points to study the physics of manganite perovskites [4]. For experimental review see [5].

[4] A.J. Millis, P.B. Littlewood, and B.I. Shraiman, Phys. Rev. Lett. 74, 5144 (1995).

Double Exchange Alone Does Not Explain the Resistivity of $\mathrm{La}_{1-x} \mathrm{Sr}_{x} \mathrm{MnO}_{3}$.

[5] A. P. Ramirez, J. Phys.: Condens. Matter 9, 8171 (1997). Colossal magnetoresistance.

[6] P. -G. de Gennes, Phys. Rev. 118, 141-154 (1960). Effects of Double Exchange in Magnetic Crystals.

[7] Eduardo Fradkin, Field Theories of Condensed Matter Systems, Redwood City, Calif.: Addison-Wesley Pub. Co., 1991.

[8] M. Berry, Proc. R. Soc. Lond. A 392, 45 (1984).

[9] S. B. Treiman, R. Jackiw, B. Zumino, and E. Witten,
Current Algebra and Anomalies, (Princeton University Press, 1985).

[10] As a general reference on integration over Grassman variables see, e.g., J. W. Negele and H. Orland, Quantum Many-Particle Systems, Perseus Pr, 1998.

[11] E. Witten, Nucl. Phys. B 223, 422 (1983). Global aspects of current algebra.

[12] Here and in the following we use brief "matrix" notations so that, e.g., this expression means actually $\frac{1}{2} c_{i \alpha}^{\dagger} \vec{\sigma}_{\alpha \beta} c_{i \beta}$ with implicit summation over repeated spin indices $\alpha, \beta=1,2$.

[13] One should of course remember that this is a dangerous thing to do because charge degrees of freedom are not gapped in infinite system and one should expect some nonlocal spin-spin interactions induced in the process of such an integration.

[14] Authors are grateful to D. A. Ivanov who attracted their attention to this fact.

[15] The target space $G$ is, in fact, homotopic to a tangent bundle to $S^{2}$ which is in turn can be contracted onto $S^{2}$.

[16] The method we used to calculate the geometric phase $\dot{a} / 2$ is known as the method of embedding in quantum field theory [1] 